\title{
Vitamin D and Spontaneous Pregnancy Loss Among Attendants of Al Zahraa University Hospital
}

\author{
Samia M.E Hamad ${ }^{1}$, Mahasen M.M.Ibrahim ${ }^{1}$, Monira M.Gad ${ }^{2}$, AsmaaS.Hassan $^{3}$, Asmaa M. Abo El Elea $^{1^{*}}$ \\ ${ }^{1}$ Public Health and CommunityMedicine, ${ }^{2}$ Obstetrics and Gynecology and \\ ${ }^{3}$ Clinical Pathology Departments, Faculty of Medicine (for Girls), Al-Azhar University \\ *Corresponding author: e-mail: drasmaa_azhar2010@yahoo.com, mobile:01025641655
}

\begin{abstract}
Background: Pregnancy loss (PL), the most common negative outcome of pregnancy, has an important emotional impact on women and their partners.

Aim of the work: This work aims to assess serum vitamin D status among pregnant females with and without abortion and to determine possible risk factors influencing vitamin D deficiency among pregnant females.

Participants and Methods: a case control study included250 pregnant women during the first 20 weeks of gestation, aged from 20 to 35 years. Cases comprised those presented with current idiopathic abortion (125), while those without current or previous abortion were selected as controls chosen from attendees of Obstetric and Gynecological Department of Al Zahraa University Hospital in Cairo, Egypt during 2017\&2018. The data were collected by completing interview questionnaire, taking anthropometric measurement (weight and height) then calculating body mass index (BMI) and taking blood sampling to assess vitamin D level.

Results:Vitamin D deficiency (VDD) and insufficiency were high among all studied pregnant women (deficient in $57.5 \%$ and $60 \%$, insufficient in $32.5 \%$ and $27.5 \%$ of cases and controls respectively) while optimal level was only among $10 \%$ of cases and $12.5 \%$ of controls with $\mathrm{OR}=1.28$ and C.I $=(0.319-5.186)$. By comparing deficient and optimal groups, it was found that obesity $(\mathrm{OR}=1.5)$, nullipara parity $(\mathrm{OR}=7.77)$, less indoor activity $(\mathrm{OR}=1.4)$ and less outdoor activity $(\mathrm{OR}=16.47)$, more fish intake $(\mathrm{OR}=19)$ and less yogurt consumption $(\mathrm{OR}=1.57)$ were possible risk factors for VDD, while urban residence, no working status, low educational level; low socio economic status, white skin color and exposure to sun with more duration and more body surface exposed were protective factors.

Conclusion:Vitamin D deficiency was more among pregnant females and it was detected as one of the possible modifiable risk factors of spontaneous pregnancy loss especially among recurrent ones.
\end{abstract}

Keywords: Spontaneous pregnancy loss, Vitamin D.

\section{INTRODUCTION}

A pregnancy loss or spontaneous abortion (SA) is defined as the spontaneousdemise of a pregnancy before the fetus reaches viability. Itincludes all pregnancy losses (PL) from the time of conception until24 weeks of gestation $^{(\mathbf{1}, \mathbf{2})}$. Approximately $15 \%$ of pregnant womenexperience sporadic loss, $2 \%$ experience 2 consecutive PL and 0.4 to $1 \%$ have 3 consecutive PL ${ }^{(3)}$. Recurrent pregnancy loss (RPL) is definedas the loss of 2 or more pregnancies; RPL affects approximately 1$2 \%$ of reproductivewomen. Causes of RPL are related to genetic factors, anatomical abnormalities, infections, and endocrine disorders ${ }^{(4)}$.

Vitamin D status during pregnancy has been drawing great attention. Today, vitamin $\mathrm{D}$ is thought to have multiple functions beyond its role(s) in bone health (due to maintaining calcium homeostasis and promoting bone mineralization ${ }^{(5)}$. Recently, vitamin D nuclear receptors (VDR) have been identified in numerous tissues including organs involved in the reproduction and infant growth such as the ovary, testis, placenta and mammary gland ${ }^{(\mathbf{8})}$.There is some evidence suggesting that it modulates human reproductive processes ${ }^{(9)}$.Also, it has significant roles in regulating cell proliferation and differentiation and modulating innate and adaptive immune responses ${ }^{\mathbf{6}}$, ${ }^{7}$.It inhibit proliferation of T helper 1 (Th1) cells and limit their production of cytokines, such as interferon gamma,interleukin-2 (IL-2) and tumor necrosis factor-alpha .Conversely, it induces T helper 2 (Th2) cytokines, such as IL-4, IL-5, IL-6.Vitamin D and also regulates $\mathrm{B}$ cell immunity. It down-regulates the proliferation and differentiation of B lymphocytes and inhibits IgG production' (10). Thus, a dominant Th2immune response is important to maintain maternal-fetal relationship for successful pregnancy. In contrast autoimmunity and dysregulated cellular immune reactions may be responsible for immunological alterations leading to recurrent pregnancy loss ${ }^{(\mathbf{1 1})}$.

Vitamin D deficiency during pregnancy is a worldwide epidemic and its prevalence ranges from $18-84 \%$, depending on the country as well as local clothing customs ${ }^{(\mathbf{1 2})}$.In Egypt, a study conducted in 2015 by Botroset al. ${ }^{(13)}$ found that $54 \%$ of 
pregnantfemales had vitamin D deficiency and 10\% had vitamin D insufficiencyand another work in 2014 carried out byEl Rifai et al. $^{(\mathbf{1 4})}$ reported that the prevalence of vitamin D deficiency and insufficiency among pregnant women reached $40 \%$ and $28.9 \%$, respectively.

There are multiple risk factors affecting hypovitaminosis among pregnant women as air pollution, high latitude, seasonal variation more in winter and autumn and the duration of sun exposure, obesity, black skin color as well as the low education level and low income of the mother ${ }^{(15)}$.

Furthermore, maternal VDD had adverse maternal and fetal outcomes, including gestational diabetes, pre-eclampsia, preterm labor, low birth weight, caesarean section and sporadic spontaneous abortion $^{(\mathbf{1 6}, \mathbf{1 7})}$. However, the relationship of vitamin D deficiency and insufficiency in the firsttrimesterpregnancy with PL or non-gravid childbearing aged women withspontaneous pregnancy loss history is less clear.

So, the aim of this work was to assess the role of vitamin $\mathrm{D}$ and abortion.

\section{STUDY HYPOTHESIS}

The null hypothesis ( $\mathrm{H} 0)$ was assumed that there is no difference in vitamin $\mathrm{D}$ levels between pregnant females with and without spontaneous PL. AIM OF THE WORK

The aim of the present work was to determine serum vitamin $\mathrm{D}$ status among pregnant females with and without abortion at Al Zahraa University Hospital as well as possible factors affecting its level among pregnant women.

\section{PATIENTS AND METHODS}

\section{Study Design:}

Acase control study was conducted on a total number of 250 participants (125 cases and 125 controls). The study was conducted at Obstetric and Gynecological Department (outpatient and inpatient clinic) of $\mathrm{Al}$ Zahraa University Hospital, Cairo during 2017 and 2018.

\section{Sampling Technique:}

\section{- Study population and type of sample:}

Two randomly selected days per week were decided for visiting the Obstetric and Gynecological Department, to recruit cases from all pregnant women presented with any type of abortion (missed,threatened) during the first 20 weeks of gestation. Controls were recruited from the pregnant females without either current or previous history of abortion and presented to the department for antenatal care.
Inclusion criteria: include all females during current abortion during the first 20 weeks of gestation, their ages between 20-35 years old with history of regular menstrual cycles and without history of hormonal or vitamin D supplementation within the last 3 months. TheInclusion criteria forselection controlsinclude pregnant women within the same age, during the same gestational age and without current or even previous history of abortion.

\section{Exclusion criteria:}

Patients suffering from rheumatoid arthritis or systemic lupus, uterine anomalies, recent acute viral infection, toxoplasmosis and chronic diseases as hypertension, DM, thyroid dysfunction or had multiple pregnancies were excluded.

\section{- Sample size:}

Sample size was calculated by using Raosoft Sample size calculator with $95 \%$ confidence level and by using population size about 1200 and prevalence of spontaneous abortion were $10 \%$.We have planned the study of matched sets of cases and controls with one matched control per case.All patients were subjected to:

\section{Research tools:}

1- Interview Questionnaire: the structured questionnaire was designed to collect demographic characteristics of the studied pregnant females, Obstetrics and gynecological history as gestational age, parity and history of previous abortion and possible risk factors affecting vitamin $\mathrm{D}$ level among pregnant females as sun exposure characteristics, skin color, sun protection use, dietary intake and physical activity.

2- Anthropometric measurements: Body weight and height were measured, and then body mass index (BMI) was calculated and according to BMI (WHO) ${ }^{(18)}$ pregnant females were classified as underweight $(\mathrm{BMI}=<18.50)$, normal weight $(\mathrm{BMI}=$ 18.50-24.99), overweight $\quad(\mathrm{BMI}=25.00-$ 29.99) and obesity (BMI $\geq 30$ ).

3- Laboratory investigation of vitamin $D$ level: Subsample (80 participants) was investigated for serum 25-hydroxyvitamin D by using enzyme immunoassay (EIA kit) KT815 with ELISA SystemAS1851Das; Italy (reader) and16041412BioTek; USA (washer) for the quantitative measurement of total $25 \mathrm{OH}$ vitamin D 2/3 level in serum. Vitamin D status was classified according to the endocrine society, the level of serum $25(\mathrm{OH})$ D was classified into: Vitamin D 
deficient if serum $25(\mathrm{OH}) \mathrm{D}$ was $(\leq 20)$ $\mathrm{ng} / \mathrm{dl}$, Vitamin $\mathbf{D}$ insufficient if serum $25(\mathrm{OH})$ was (21-29) $\mathrm{ng} / \mathrm{dl}$ and Vitamin D sufficient if serum $25(\mathrm{OH})$ was $(30-100)$ $\mathrm{ng} / \mathrm{dl}$.

\section{Statistical analysis}

Pre-coded data were statistically analyzed using SPSS 20. For descriptive purpose, qualitative data were presented as frequencies and percentages. Mean, standard deviations and ranges were used to describe quantitative numeric variables.

To assess the significance in the observed differences between cases and controls, Pearson Chi square- test $\left(\mathrm{X}^{2}\right)$ for independence was used for qualitative data and Fishers Exact test was used instead for cells less than 5. The Student's independent t-test was used for the differences between means of two continuous variables of unpaired group. The significance level was taken at 0.05 and $95 \%$ confidence limit. The results were deemed to be statistically significant if the $p$ value (two tallied) was $\leq 0.05$. Odds ratios (OR) is the preferred measure of association.

The studied group was classified into two groups to assess factors affecting vitamin D level by comparing two groups who were: vitamin D -deficient andvitamin D- optimal.

\section{Ethical consideration:}

The ethical committee at Al-Azhar Faculty of Medicine for Girls, approved the research, followed by an ethical permission of Al-Zahraa University Hospital and informed oral consent was obtained from all patients.

\section{RESULTS}

The mean age of cases and controls were $(27.54 \pm 4.92$ and $25.58 \pm 4.3)$ years respectively, while regarding age group, those whose age ranged between 19 and 24 years represent 31.2 $\%$ of cases and $45.6 \%$ of controls while those whose age ranged between 30-35 years formed $40 \%$ of cases and $2.4 \%$ of controls. Also, $85.6 \%$ of cases and $62.4 \%$ of controls were residing urban areas and $75.2 \%$ of cases and $88.8 \%$ of controls were housewives. Those who complete high level of education (university and post grade) were more in cases than in controls $(39 \%$ in cases compared to $20.8 \%$ in controls) while $40.8 \%$ of cases complete secondary education in comparison to $53.6 \%$ of controls withstatistically significant difference (pvalue $\leq 0.05$ ). In addition, $62.4 \%$ of cases and $66.4 \%$ of controls were belonging to middle social class.

As regard body mass index (BMI), overweight females represented $33.2 \%$ of cases and $33.6 \%$ of controls while obese formed $30.4 \%$ of cases and $40 \%$ of controls.

Table (1) shows that the mean gestational age was 10.78 weeks \pm 4.37 for cases and 19.18 weeks \pm 2.42 for controls with statistically significant difference between groups, $(\mathrm{P}$ value $\leq 0.05)$.Regarding parity, the women who had never given birth assumed nullipara and formed $29.6 \%$ of cases and $36.8 \%$ of controls while those who gave birth three times and more represent $18.4 \%$ of cases and $7.2 \%$ of controls. Nearly one quarter of cases $(25.6 \%)$ had a history of previous abortion with $68.7 \%$ of them occurring before two months of gestation and those who experienced recurrent abortion $(\geq 2)$ represented $62.5 \%$ of those who reported that they had abortion before, Figure (1).

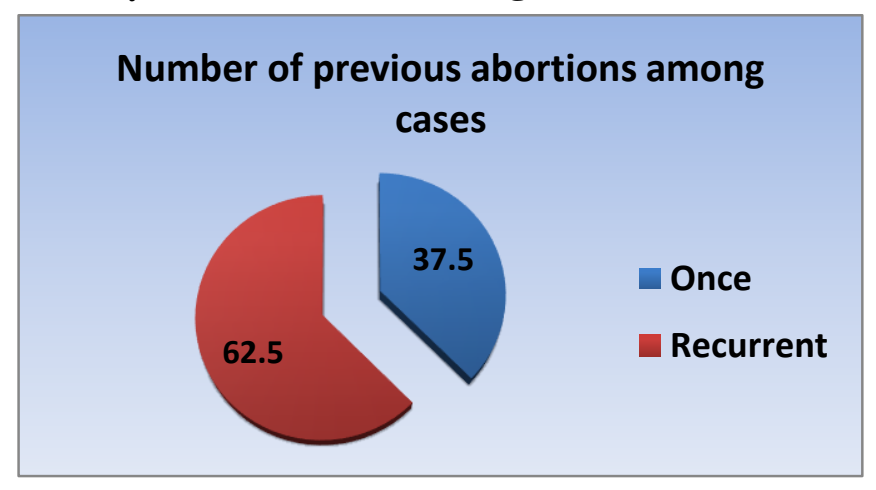

Figure (1): Number of previous abortions among cases.

Table (2) demonstrates that the serum vitamin $\mathrm{D}$ level among cases ranged from 6.61 $\mathrm{ng} / \mathrm{mL}$ to $34.1 \mathrm{ng} / \mathrm{mL}$ and its mean $\pm \mathrm{SD}$ was $(19.84 \mathrm{ng} / \mathrm{mL} \pm 7.04)$ in comparison to controls whose vitamin D ranged from $11.6 \mathrm{ng} / \mathrm{mL}$ to 37.8 $\mathrm{ng} / \mathrm{mL}$ and its mean $\pm \mathrm{SD}$ was $20.19 \mathrm{ng} / \mathrm{mL} \pm$ 7.48.from these figures and according to the Endocrine Society cut-offs for serum vitamin D level, vitamin D was deficient among $57.5 \%$ of cases and $60 \%$ of controls and was insufficient among $32.5 \%$ and $27.5 \%$ of cases and controls respectively while it was normal among $10 \%$ of cases and $12.5 \%$ of controls with $\mathbf{O R}=1.28$ and $\mathbf{C . I}=(0.319-5.186)$ without any statistical significant difference between groups, ( $\mathrm{P}$ value $>0.05)$. 


\begin{tabular}{|c|c|c|c|c|c|}
\hline Items Groups & \multicolumn{2}{|c|}{$\begin{array}{c}\text { Case } \\
\text { (Current Abortion) } \\
(125) \\
\end{array}$} & \multicolumn{2}{|c|}{$\begin{array}{c}\text { Control } \\
\text { (Current Pregnancy) } \\
(125) \\
\end{array}$} & Test of significance \\
\hline $\begin{array}{ll}\text { Gestational age: } \\
\circ & \text { Range } \\
\circ & \text { Mean } \pm \mathrm{SD}\end{array}$ & \multicolumn{2}{|c|}{$\begin{array}{c}(3-20) \\
10.78 \pm 4.37\end{array}$} & \multicolumn{2}{|c|}{$\begin{array}{c}(9-20) \\
19.18 \pm 2.42\end{array}$} & $\begin{array}{l}\mathbf{t}=21.293 \\
\mathbf{P}=0.000^{*}\end{array}$ \\
\hline Parity: & No $=125$ & $(\%)$ & No $=125$ & $(\%)$ & \\
\hline $\begin{array}{ll}\circ & \text { Nullipara } \\
\circ & \text { Para 1 } \\
\circ & \text { Para 2 } \\
\circ & \text { Para } \geq 3\end{array}$ & $\begin{array}{l}37 \\
36 \\
29 \\
23\end{array}$ & $\begin{array}{l}29.6 \\
28.8 \\
23.2 \\
18.4\end{array}$ & $\begin{array}{c}46 \\
39 \\
31 \\
9\end{array}$ & $\begin{array}{c}36.8 \\
31.2 \\
24.8 \\
7.2\end{array}$ & $\begin{array}{l}\mathbf{X}^{2}=7.288 \\
\mathbf{P}=0.063\end{array}$ \\
\hline $\begin{array}{l}\text { Previous abortion: } \\
\circ \text { Yes } \\
\circ \text { No } \\
\end{array}$ & $\begin{array}{l}32 \\
93\end{array}$ & $\begin{array}{l}25.6 \\
74.4\end{array}$ & $\begin{array}{c}0 \\
125\end{array}$ & $\begin{array}{c}0 \\
100\end{array}$ & \\
\hline $\begin{array}{l}\text { Number of previous } \\
\text { abortions: } \\
\circ \quad \text { Once } \\
\circ \quad \text { Recurrent }(\geq 2)\end{array}$ & $\begin{array}{l}12 \\
20\end{array}$ & $\begin{array}{l}37.5 \\
62.5\end{array}$ & $\begin{array}{l}0 \\
0\end{array}$ & $\begin{array}{l}0 \\
0\end{array}$ & \\
\hline $\begin{array}{l}\text { Gestational age of previou } \\
\text { abortion: } \\
\circ \text { Before two months. } \\
\circ \text { After two months. }\end{array}$ & $\begin{array}{l}22 \\
10\end{array}$ & $\begin{array}{l}68.7 \\
31.3\end{array}$ & $\begin{array}{l}0 \\
0\end{array}$ & $\begin{array}{l}0 \\
0\end{array}$ & \\
\hline
\end{tabular}

*Statistically significant difference, $(\mathrm{P}$ value $\leq 0.05)$.

Table (3) shows that $87.3 \%$ of deficient population and $88.9 \%$ of optimal population were housewives with $\mathbf{O R}=0.861$ C.I: $(0.096-7.719)$ while those who resided urban areas formed $62 \%$ of deficient population and $66.7 \%$ of optimal population with $\mathbf{O R}=0.815$ C.I: $(0.188-3.531)$. Regarding education, mostly of deficient population (93\%) was educated compared to $88.9 \%$ of optimal population with $\mathbf{O R}=0.606$ C.I: $(0.063-5.86)$. Also, nearly three quarters $(73.2 \%)$ of deficient population and $88.9 \%$ of optimal ones were belonging to low socio-economic status with $\mathbf{O R}=0.342$ C.I: $(0.040-2.92)$. it is clear that $49.3 \%$ of deficient one and $11.1 \%$ of optimal group were nullipara with $\mathbf{O R}=7.77$ C.I: $(0.924-65.46)$ with statistical significance difference, (pvalue $\leq 0.05)$.Figure (2).

Table (2):Serum Vitamin D level among studied sub sample of cases and controls and its association with spontaneous pregnancy loss

\begin{tabular}{|c|c|c|c|c|c|}
\hline $\begin{array}{l}\text { Groups } \\
\text { Items }\end{array}$ & \multicolumn{2}{|c|}{$\begin{array}{c}\text { Case } \\
\text { (Current Abortion) }\end{array}$} & \multicolumn{2}{|c|}{$\begin{array}{c}\text { Control } \\
\text { (Current Pregnancy) }\end{array}$} & Test of significance \\
\hline & $\mathrm{No}=40$ & $\%$ & $\mathrm{No}=40$ & $\%$ & \multirow[b]{2}{*}{$\begin{array}{l}\text { Fisher's exact }=0.361 \\
\text { Exact sig. } \mathbf{P}=0.898\end{array}$} \\
\hline $\begin{array}{cl}\text { Vitamin D level }(\mathbf{n g} / \mathbf{m L}) \\
\circ & \text { Deficiency }(\leq 20) \\
\circ & \text { Insufficient }(21-29) \\
\circ & \text { Normal }(30-100)\end{array}$ & $\begin{array}{c}23 \\
13 \\
4\end{array}$ & $\begin{array}{c}57.5 \\
32.5 \\
10 \\
\end{array}$ & $\begin{array}{c}24 \\
11 \\
5\end{array}$ & $\begin{array}{c}60 \\
27.5 \\
12.5 \\
\end{array}$ & \\
\hline $\begin{array}{l}\text { Vitamin D level }(\mathbf{n g} / \mathbf{m L}) \\
\text { O } \\
\text { Deficient/insufficient }(<30) \\
0\end{array}$ & $\begin{array}{c}36 \\
4\end{array}$ & $\begin{array}{l}90 \\
10\end{array}$ & $\begin{array}{c}35 \\
5\end{array}$ & $\begin{array}{l}87.5 \\
12.5\end{array}$ & $\begin{aligned} & \mathbf{X}^{\mathbf{2}}=0.125 \\
& \mathbf{O R}=1.28 \\
& \mathbf{C} . \mathbf{I}=(0.319-5.186) \\
& \mathbf{P}=0.723\end{aligned}$ \\
\hline $\begin{array}{l}\text { Vitamin D level }(\mathbf{n g} / \mathbf{m L}) \\
\circ \quad \text { Mean } \pm S D\end{array}$ & \multicolumn{2}{|c|}{$19.84 \pm 4.04$} & \multicolumn{2}{|c|}{$20.19 \pm 4.48$} & $\begin{array}{r}\mathbf{t}=0.212 \\
\mathbf{P}=0.833\end{array}$ \\
\hline
\end{tabular}




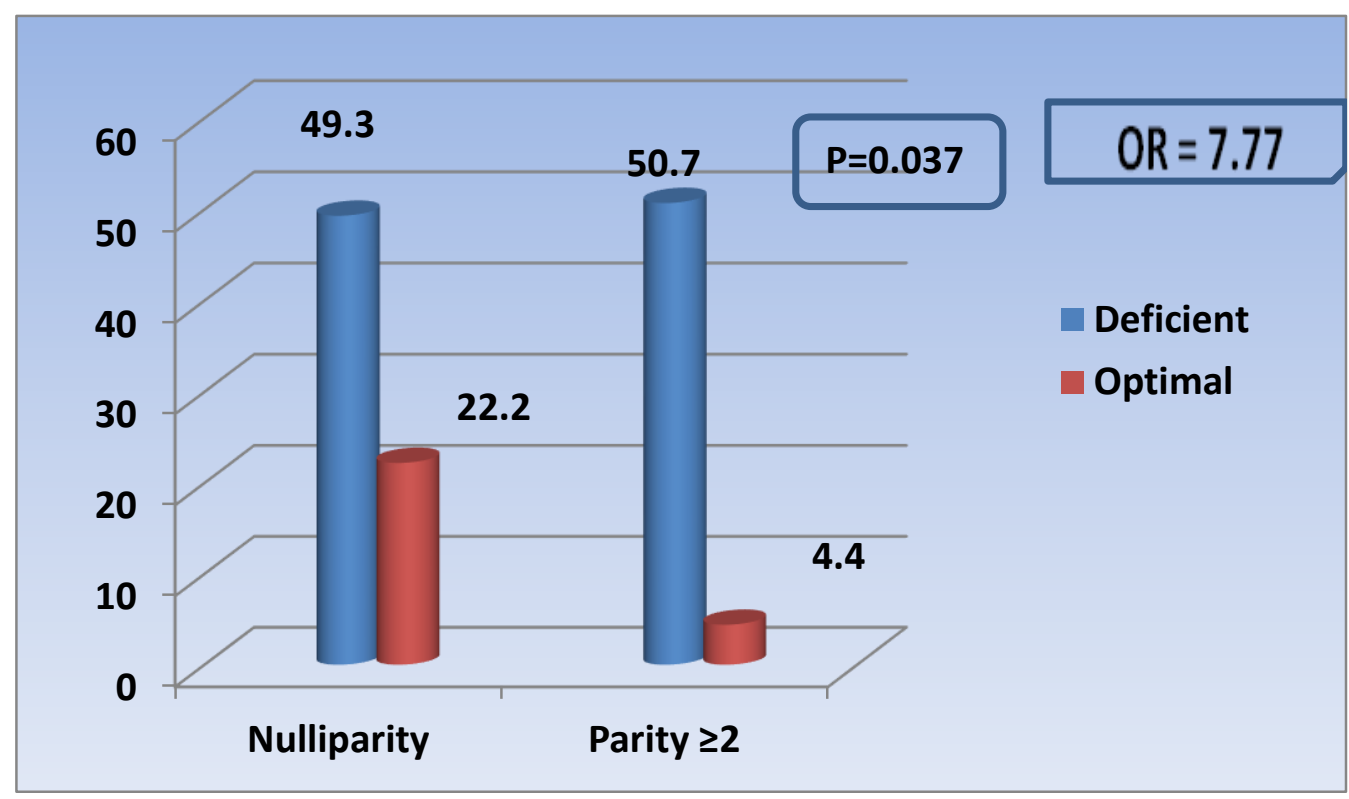

Figure (2): Vitamin D level and parity among studied subsample of cases and controls

Table (3): The association between socio demographic and parity of studied sub sample and vitamin D status.

\begin{tabular}{|c|c|c|c|c|c|c|}
\hline \multirow{3}{*}{ Items } & \multicolumn{4}{|c|}{ Vitamin D level } & \multirow[t]{3}{*}{ Test of significance } & \multirow{3}{*}{$\begin{array}{c}\text { Exact sig. } \\
\text { P-value }\end{array}$} \\
\hline & \multicolumn{2}{|c|}{$\begin{array}{c}\text { Deficient } \\
(\leq 29 \mathrm{ng} / \mathrm{mL})\end{array}$} & \multicolumn{2}{|c|}{$\begin{array}{c}\text { Optimal } \\
(30-100 \mathrm{ng} / \mathrm{mL}) \\
\end{array}$} & & \\
\hline & $\mathrm{No}=71$ & $\%$ & $\mathrm{No}=9$ & $\%$ & & \\
\hline $\begin{array}{cl}\text { Occupation: - } \\
\circ & \text { Housewife } \\
\circ & \text { Employed }\end{array}$ & $\begin{array}{c}62 \\
9\end{array}$ & $\begin{array}{l}87.3 \\
12.7\end{array}$ & $\begin{array}{l}8 \\
1\end{array}$ & $\begin{array}{l}88.9 \\
11.1\end{array}$ & $\begin{array}{c}\mathbf{X}^{\mathbf{2}}=0.18 \\
\mathbf{O R}=0.861 \\
\text { C.I: }(0.096-7.719)\end{array}$ & 1.000 \\
\hline $\begin{array}{c}\text { Residence: - } \\
\circ \text { Urban } \\
\circ \text { Rural }\end{array}$ & $\begin{array}{l}44 \\
27\end{array}$ & $\begin{array}{l}62 \\
38\end{array}$ & $\begin{array}{l}6 \\
3\end{array}$ & $\begin{array}{l}66.7 \\
33.3\end{array}$ & $\begin{array}{c}\mathbf{X}^{\mathbf{2}}=0.075 \\
\mathbf{O R}=0.815 \\
\text { C.I: }(0.188-3.531)\end{array}$ & 1.000 \\
\hline \begin{tabular}{ll}
\multicolumn{2}{l}{ Education } \\
$\circ$ & Low educated \\
$\circ$ & High educated
\end{tabular} & $\begin{array}{c}5 \\
66\end{array}$ & $\begin{array}{c}7 \\
93\end{array}$ & $\begin{array}{l}1 \\
8\end{array}$ & $\begin{array}{l}11.1 \\
88.9\end{array}$ & $\begin{array}{c}\mathbf{X}^{2}=0.191 \\
\mathbf{O R}=0.606 \\
\text { C.I: }(0.063-5.86)\end{array}$ & 1.000 \\
\hline $\begin{array}{ll}\text { Socio Economic Status: } \\
- \\
\circ \text { Low } \\
\circ \text { High }\end{array}$ & $\begin{array}{l}52 \\
19\end{array}$ & $\begin{array}{l}73.2 \\
26.8\end{array}$ & $\begin{array}{l}8 \\
1\end{array}$ & $\begin{array}{l}88.9 \\
11.1\end{array}$ & $\begin{array}{c}\mathbf{X}^{2}=1.043 \\
\mathbf{O R}=0.342 \\
\text { C.I: }(0.040-2.92)\end{array}$ & 0.437 \\
\hline
\end{tabular}




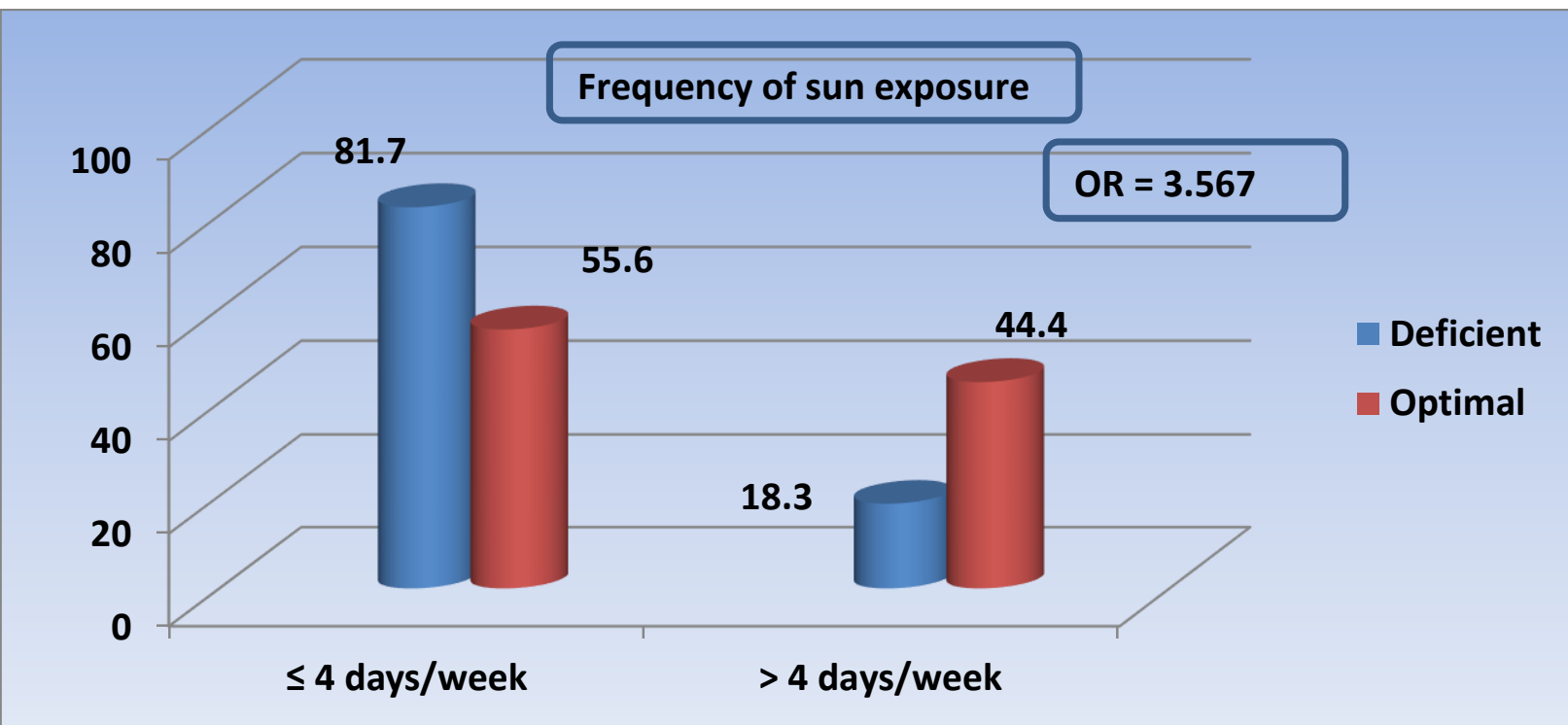

Figure (3): Association of frequency of Sun exposure as regardvitamin Dstatus among studied subsample of cases and controls.

Figure (3) shows that those who exposed to the sun four day and less represented $81.7 \%$ for deficient ones and 55.6\% for optimal ones with OR=3.567C.I: (0.84- 15.15). While Figure (4) clarifies that $29.6 \%$ of deficient group was obese compare $\mathrm{d}$ to $22.2 \%$ of optimal ones with $\mathbf{O R}=1.5$ C.I: $(0.282-7.67)$.

Table (4): The association between sunning practice of studied sup sample and vitamin D level.

\begin{tabular}{|c|c|c|c|c|c|c|}
\hline \multirow{3}{*}{ Items Groups } & \multicolumn{4}{|c|}{ Vitamin D level } & \multirow{3}{*}{ Test of significance } & \multirow{3}{*}{$\begin{array}{l}\text { Exact sig. } \\
\text { P-value }\end{array}$} \\
\hline & \multicolumn{2}{|c|}{$\begin{array}{c}\text { Deficient } \\
(\leq 29 \mathrm{ng} / \mathrm{mL}) \\
\end{array}$} & \multicolumn{2}{|c|}{$\begin{array}{c}\text { Optimal } \\
(30-100 \mathrm{ng} / \mathrm{mL})\end{array}$} & & \\
\hline & $\mathrm{No}=71$ & $\%$ & $\mathrm{No}=9$ & $\%$ & & \\
\hline \begin{tabular}{cc}
\multicolumn{2}{c}{ Skin color } \\
$\circ$ & White \\
$\circ$ & Black \\
\end{tabular} & $\begin{array}{l}29 \\
42\end{array}$ & $\begin{array}{l}40.8 \\
59.2\end{array}$ & $\begin{array}{l}4 \\
5\end{array}$ & $\begin{array}{l}44.4 \\
55.6\end{array}$ & $\begin{array}{c}\mathbf{X}^{2}=0.043 \\
\mathbf{O R}==0.863 \\
\text { C.I: }(0.213-3.491) \\
\end{array}$ & 1.000 \\
\hline $\begin{array}{l}\text { Body parts exposed: } \\
\circ \text { Face and hand } \\
\bigcirc \text { Hand only }\end{array}$ & $\begin{array}{c}66 \\
5\end{array}$ & $\begin{array}{c}93 \\
7\end{array}$ & $\begin{array}{l}9 \\
0\end{array}$ & $\begin{array}{c}100 \\
0\end{array}$ & $\begin{array}{l}\mathbf{X}^{2}=0.676 \\
\mathbf{O R}==0.88 \\
\text { C.I: }(0.809-0.957)\end{array}$ & 0.636 \\
\hline $\begin{array}{cl}\text { Score of Sun Protection Use: } \\
\circ & 4 \text { forms } \\
\circ & 5 \text { forms }\end{array}$ & $\begin{array}{l}44 \\
27\end{array}$ & $\begin{array}{l}62 \\
38 \\
\end{array}$ & $\begin{array}{l}5 \\
4\end{array}$ & $\begin{array}{l}55.6 \\
44.4\end{array}$ & $\begin{array}{c}\mathbf{X}^{2}=0.139 \\
\mathbf{O R}=1.3 \\
\text { C.I: }(0.322-5.283) \\
\end{array}$ & 0.729 \\
\hline $\begin{array}{l}\text { Time of sun exposure: } \\
\circ \quad \text { Around midday (10 am- } \\
\text { 3pm). } \\
\circ \quad \text { Away from midday. }\end{array}$ & 47 & 66.2 & $\overline{5}$ & 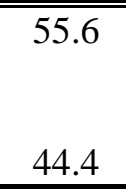 & $\begin{array}{c}\mathbf{X}^{\mathbf{2}}=0.398 \\
\mathbf{O R}==1.567 \\
\text { C.I: }(0.38-6.37)\end{array}$ & 0.712 \\
\hline $\begin{array}{l}\text { Adequacy of sun exposure } \\
\text { duration: } \\
\circ \text { In adequate. } \\
\circ \text { Adequate. }\end{array}$ & $\begin{array}{l}35 \\
36\end{array}$ & $\begin{array}{r}49.3 \\
50.7 \\
\end{array}$ & $\begin{array}{l}5 \\
4 \\
\end{array}$ & $\begin{array}{r}55.6 \\
44.4\end{array}$ & $\begin{array}{c}\mathbf{X}^{2}=0.125 \\
\mathbf{O R}==0.778 \\
\text { C.I: }(0.193-3.137)\end{array}$ & 1.00 \\
\hline
\end{tabular}

*Significant level (pvalue $\leq 0.05$ ).

Table (4) clarifies thatthose who were belonging to black skin color represented $59.2 \%$ of deficient group compared to $55.6 \%$ of optimal ones with $\mathbf{O R}=0.863$ C.I: $(0.213-3.491)$. Concerning to sun exposure, majority of participant exposing face and hand to the sun represented $93 \%$ and $100 \%$ for deficient and optimal ones respectively with $\mathbf{O R}==0.88$ and C.I: $(0.809-0.957)$. Also, those who exposed to the sun around midday (10 am- 
$3 \mathrm{pm}$ ) represented $66.2 \%$ of deficient population compared to $55.6 \%$ of optimal ones with $\mathbf{O R}==1.567$ C.I: (0.38- 6.37). Also, this table shows that duration of sun exposure was adequate in $50.7 \%$ of deficient ones and $44.4 \%$ of optimal group, while $44.4 \%$ of optimal ones used 5 forms of sun protection clothing that used outdoor to protect themselves from the sun in comparison with $38 \%$ of deficient group.

Table (5): The association between dietary sources of vitamin D among sub studied sample and their Vitamin D level.

\begin{tabular}{|c|c|c|c|c|c|c|}
\hline \multirow{3}{*}{ Items Groups } & \multicolumn{4}{|c|}{ Vitamin D level } & \multirow[t]{3}{*}{ Test of significance } & \multirow{3}{*}{$\begin{array}{c}\text { Exact sig. } \\
\text { P-value }\end{array}$} \\
\hline & \multicolumn{2}{|c|}{$\begin{array}{c}\text { Deficient } \\
(\leq 29 \mathrm{ng} / \mathrm{mL})\end{array}$} & \multicolumn{2}{|c|}{$\begin{array}{c}\text { Optimal } \\
(30-100 \mathrm{ng} / \mathrm{mL})\end{array}$} & & \\
\hline & $\mathrm{No}=71$ & $\%$ & $\mathrm{No}=9$ & $\%$ & & \\
\hline 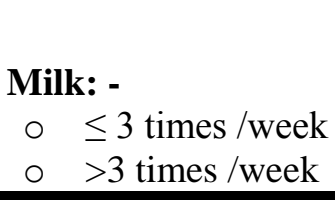 & $\begin{array}{l}30 \\
41\end{array}$ & $\begin{array}{l}42.3 \\
57.7\end{array}$ & $\begin{array}{l}4 \\
5\end{array}$ & $\begin{array}{l}44.4 \\
55.6\end{array}$ & $\begin{array}{c}\mathbf{X}^{2}=0.016 \\
\mathbf{O R}=0.915 \\
\text { C.I: }(0.226-3.696)\end{array}$ & 1.000 \\
\hline $\begin{array}{l}\text { Yogurt: - } \\
\circ \quad \leq 3 \text { times /week } \\
\circ>3 \text { times /week }\end{array}$ & $\begin{array}{l}22 \\
49\end{array}$ & $\begin{array}{l}31 \\
69\end{array}$ & $\begin{array}{l}2 \\
7\end{array}$ & $\begin{array}{l}22.2 \\
77.8\end{array}$ & $\begin{array}{c}\quad \mathbf{X}^{2}=0.292 \\
\mathbf{O R}=1.57 \\
\text { C.I: }(0.302-8.182)\end{array}$ & 0.456 \\
\hline $\begin{array}{l}\text { Cheese: - } \\
\begin{array}{c}0 \\
0 \\
0\end{array} \text { >3 times / week /week }\end{array}$ & $\begin{array}{l}42 \\
29\end{array}$ & $\begin{array}{l}59.2 \\
40.8\end{array}$ & $\begin{array}{l}6 \\
3\end{array}$ & $\begin{array}{l}66.7 \\
33.3\end{array}$ & $\begin{array}{c}\mathbf{X}^{\mathbf{2}}=0.188 \\
\mathbf{O R}=0.724 \\
\text { C.I: }(0.167-3.132)\end{array}$ & 0.734 \\
\hline $\begin{array}{l}\text { Egg: - } \\
\begin{array}{c}\leq 3 \text { times /week } \\
0>3 \text { times /week }\end{array}\end{array}$ & $\begin{array}{l}36 \\
35\end{array}$ & $\begin{array}{l}50.7 \\
49.3\end{array}$ & $\begin{array}{l}6 \\
3\end{array}$ & $\begin{array}{l}66.7 \\
33.3\end{array}$ & $\begin{array}{c}\mathbf{X}^{2}=0.816 \\
\mathbf{O R}=0.514 \\
\text { C.I: }(0.119-2.219)\end{array}$ & 0.487 \\
\hline $\begin{array}{ll}\text { Oily } & \text { Fish: } \\
\circ & \text { Weekly } \\
\circ & \text { Monthly }\end{array}$ & $\begin{array}{l}50 \\
21\end{array}$ & $\begin{array}{l}70.4 \\
29.6\end{array}$ & $\begin{array}{l}1 \\
8\end{array}$ & $\begin{array}{l}11.1 \\
88.9\end{array}$ & $\begin{array}{c}\mathbf{X}^{\mathbf{2}}=12.159 \\
\mathbf{O R}=19.048 \\
\text { C.I: }(2.240-164.9)\end{array}$ & $0.001 *$ \\
\hline $\begin{array}{cl}\text { Cooked liver: } \\
\circ & \text { Weekly } \\
\circ & \text { Monthly }\end{array}$ & $\begin{array}{c}5 \\
66\end{array}$ & $\begin{array}{c}7 \\
93\end{array}$ & $\begin{array}{l}2 \\
7\end{array}$ & $\begin{array}{l}22.2 \\
77.8\end{array}$ & $\begin{array}{c}\mathbf{X}^{\mathbf{2}}=2.305 \\
\mathbf{O R}==0.265 \\
\text { C.I: }(.043-1.629)\end{array}$ & 0.176 \\
\hline
\end{tabular}

*Significant level (p.value $\leq 0.05)$.

Table (5) demonstrates that milk consumption was nearly similar between the two groups with $\mathbf{O R}=$ 0.915 C.I: (0.226-3.696). Also, those who consumed yogurt more than three times per week represented $69 \%$ of deficient group compared to $77.8 \%$ of optimal ones with $\mathbf{O R}=1.57$ C.I: $(0.302-8.182)$. Regarding oily fish consumption, $70.4 \%$ of deficient ones consumed once per week compared to $11.1 \%$ of optimal ones with $\mathbf{O R}=$ 19.048 C.I : (2.240-164.9) with statistically significant difference $(\mathrm{P} \leq 0.05)$. While, those who consumed cooked liver monthly among deficient ones were $66 \%$ compared to $77.8 \%$ of optimal group with $\mathbf{O R}==0.265$ C.I: $(.043$ 1.629).

Table (6) shows thatabout one third of those (33.8\%) with vitamin D deficiency had moderate indoor activity compared to all those with optimal vitamin D with $\mathbf{O R}=1.4$ C.I: $(1.116-1.694)$, While $4.2 \%$ of deficient participants had moderate outdoor activity versus to $22.2 \%$ of optimal ones with $\mathbf{O R}=6.47$ C.I: $\quad(0.920-45.5)$, with statistical significant difference between deficient and optimal groups, (pvalue $\leq 0.05$ ). 
Table (6): The association between physical activity of studied sub sample and vitamin D status

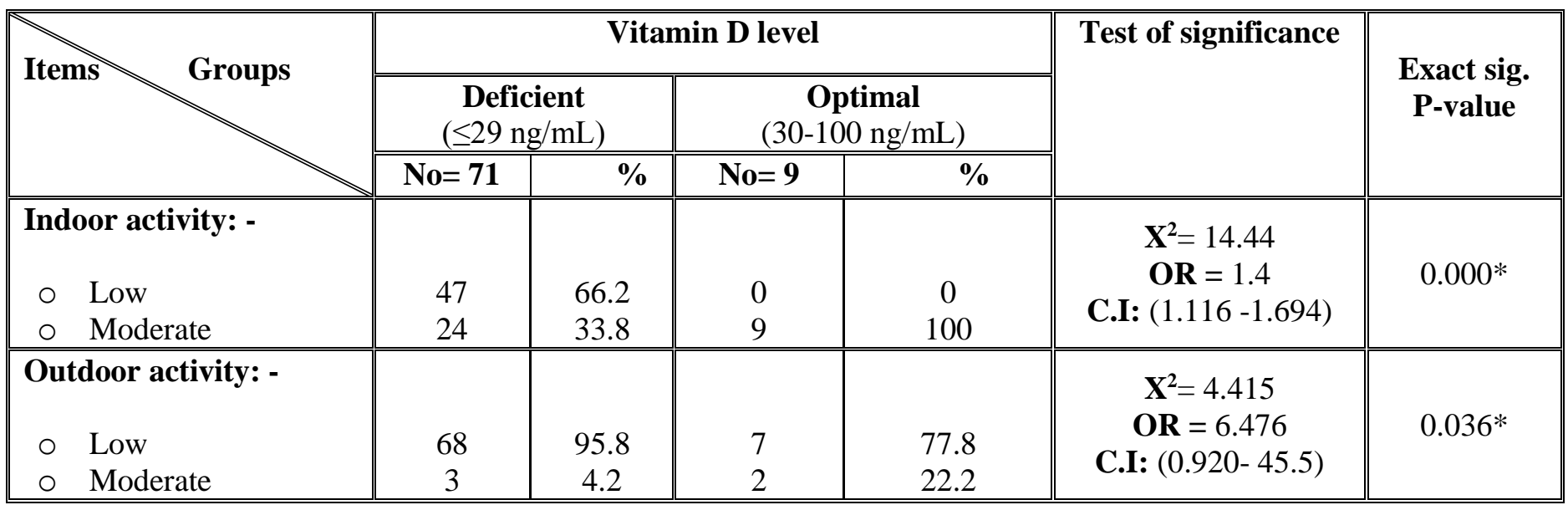

*Significant level (pvalue $\leq 0.05$ ).

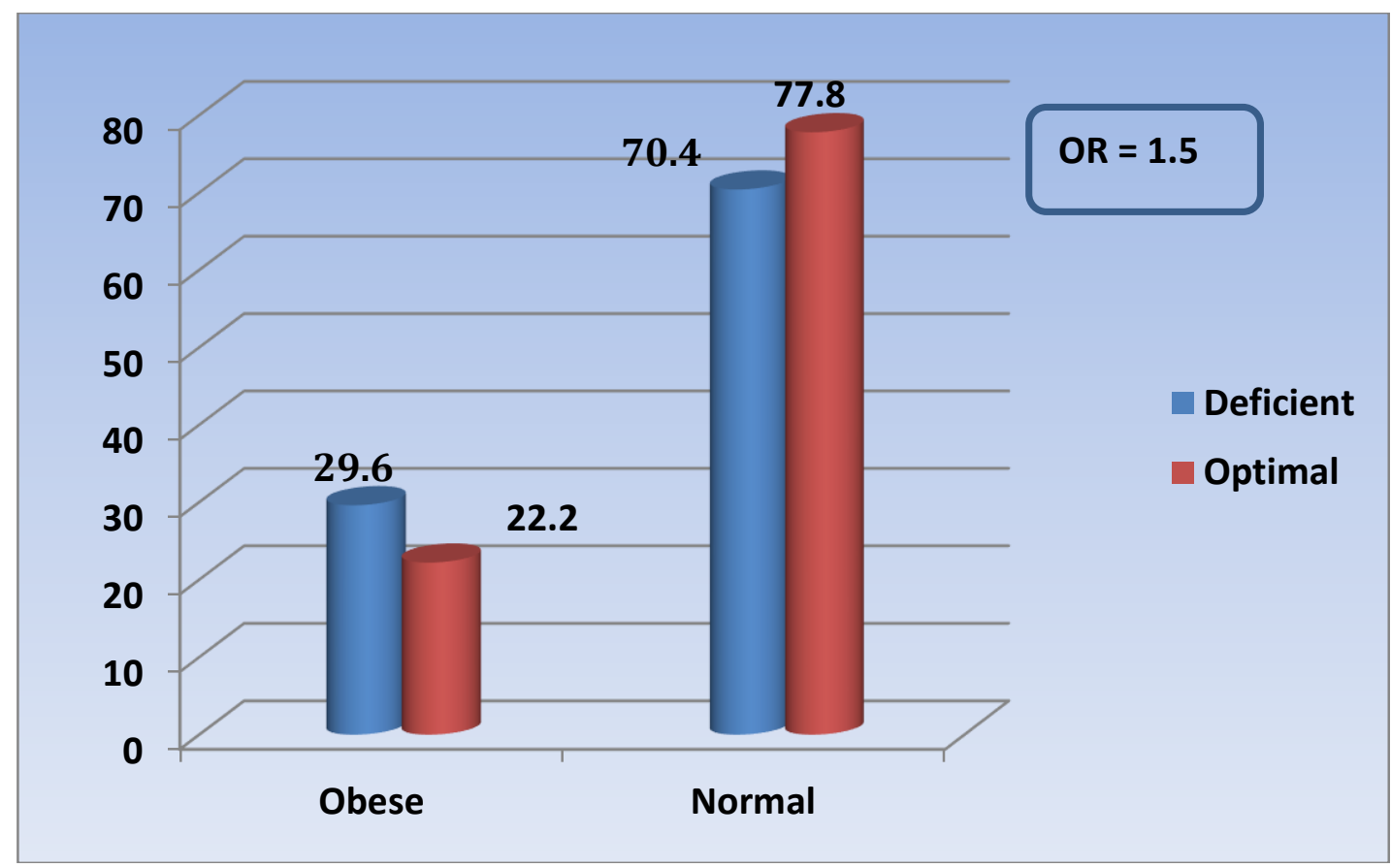

Figure (4):Body Mass Index among studied subsample of cases and controls and vitamin D level.

\section{DISCUSSION}

The current study was conducted to delineate the possible relation between vitamin $\mathrm{D}$ status and the unfavorable pregnancy outcome, the spontaneous pregnancy loss. Our data revealed that $57.5 \%$ of cases and $60 \%$ of control were deficient in vitamin $\mathrm{D}, 32.5 \%$ of cases and $27.5 \%$ of control had insufficient level, while only $10 \%$ of cases and $12.5 \%$ of control had optimal level of vitamin D. This was in accordance to what was found by Botroset al. ${ }^{(13)}$ who conducted a study in Egypt including healthy females of different age groups in Cairo and Port Said and revealed that $54 \%$ of pregnant females had vitamin D deficient and $10 \%$ had vitamin D insufficiency.Also, El Rifai et al. ${ }^{(14)}$ reported that theprevalence of vitamin D deficiency and insufficiency among pregnant women were $40 \%$ and $28.9 \%$, respectively. Furthermore, ElKoumi et al. ${ }^{(19)}$ revealed high prevalence of vitamin D deficiency among pregnant females.

In addition, Hussein et al. $^{(\mathbf{2 0 )}}$ documented from a study conducted at 12 districts within the UAE, that $(69 \%)$ of women had vitamin D deficiency, while $(22.6 \%)$ had vitamin D insufficiency with (8\%) subjects with vitamin D status of adequate status. Also, these results were supported by Woon et al. ${ }^{(21)}$ and Aji et al. ${ }^{(16)}$.

Regardingthe association ofvitamin D deficiency and spontaneous pregnancy loss, the present study reported that vitamin D deficiency was 
more among cases (women with current abortion) than control (normal pregnant women) with OR $=1.28$ and C.I $=(0.319-5.186)$ which indicated the risk of VDD in developing spontaneous pregnancy loss. These finding agreed with Gonçalves et al. ${ }^{(17)}$ whosystematically reviewed articles studied women with 2 or more spontaneous abortion and its association with VDD and found eleven Studies reported a high prevalence of VD insufficiency (VDI) or VDD in women with recurrent pregnancy loss (RPL). Also, Hou et al. (22) found thatthe regression analyses showed that PL was significantly inversely correlated with $25(\mathrm{OH}) \mathrm{D}(\mathrm{P}<0.01)$ and there was a strong association between low vitamin D levels and PL $(\mathrm{OR}=1.71$; 95\% C.I: $1.2-2.4$, P0.001).Also, Ghaedi et al. ${ }^{(23)}$ and Andersen et al. ${ }^{(24)}$ supported this association. Ozkan et al. (25) documentedthat the follicular fluid $25(\mathrm{OH}) \mathrm{D}$ levels are independent predictors of successful clinical pregnancy following in vitro fertilization; however, serum and follicular fluid levels of $25(\mathrm{OH}) \mathrm{D}$ are highly correlated.

On the other hand, Moller et al. ${ }^{(26)}$ and Flood-Nicholset al. ${ }^{(27)}$ did not find any association between vitamin $\mathrm{D}$ deficiency and the clinical outcome of miscarriage in the first-trimester pregnancy. This difference may be explained not only by differences in study design, but also by differences in our study population relative to other published studies, including the difference in maternal age, sample size and gestational age of specimen collection.

Concerning the possible effect of parity on vitamin D status, the current study revealed that nulliparouswomenwere more among those who were deficient in vitamin D (49.3\%) than those with normal level (11.1\%) with odds ratio $(\mathrm{OR}=7.77)$. These results lie in line with Aji et al ${ }^{(16)}$ who found thatpregnantwomen with nulliparous parity status had an eight times higher risk of developing VDD (OR: 7.634, CI 95\% 1.550-37.608) $(\mathrm{p}$ value $=0.012)$. Also, Perezetal. ${ }^{(28)}$ Found that nulliparous women (OR: 2.47; $\mathrm{p}=0.002$ ) related to deficient 25(OH)D levels. On contrary, Andersen et al. ${ }^{(29)}$ reported that parity was strongly and consistently inversely correlated with vitamin D levels irrespective of season. This difference may be explained as multipara women had multivitamin supplements with previous pregnancies and had experience in nutritional education in comparison with nullipara parity, while Woon et al. ${ }^{(21)}$, Ekeroma et al. ${ }^{(30)}$ and El Rifaiet al. ${ }^{(14)}$ showed that there were no associations between parity with vitamin D deficiency.

As vitamin D sources are obtained mainly from three sources, sunlight, diet, and supplementation, the present study found that vitamin $\mathrm{D}$ deficiency was more among those with less sun exposure asall optimal group exposes face and hand to the sun with $55.6 \%$ of them exposed around midday (between 10 am and $3 \mathrm{pm}$ ) compared to $93 \%$ and $66.2 \%$ of the deficient one. Although the deficient group exposed to the suitable time of sun exposure, they attained low level of vitamin D as they were exposed while they covering all the body except face and hand or hand only and the score of sun protection clothing that used outdoor to protect themselves from the sun (4 forms) were $62 \%$ and (5 forms) were $38 \%$.

Moreover, it was documented that nearly one third $(31 \%)$ of women deficient in vitamin Dconsumed yogurt three times or less per week in comparison to $22.2 \%$ of the optimal ones with odds ratio $(\mathrm{OR}=1.57)$.In addition, among deficient study population $70.4 \%$ of consumed fish weekly compared to $11.1 \%$ of the optimal ones with odds ratio $(\mathrm{OR}=19)$ with statistical significant difference. It may be due to consumption the type of fish that is poor in vitamin $\mathrm{D}$ as fish rich in vitamin $\mathrm{D}$ is expensive and not easily available.These results were supported by El Rifai et al ${ }^{\text {(14) }}$ who reported that maternal vitamin D levels showed significant correlations with skin exposure to sun and Aji et al. ${ }^{(16)}$ documentedthat there wasa positive correlation between sun exposure and vitamin D status and most of the women who had less exposure to sunlight were vitamin $\mathrm{D}$ deficient.

On the contrary, Bukhary et al. ${ }^{(31)}$ reported that there was no association between sun exposure and vitamin D levels. Furthermore, El Rifai et al. ${ }^{(14)}$ and Ekeroma et al. ${ }^{(30)}$ reported that maternal vitamin D levels were not significantly correlated with eggs and dairy products consumption. This may be due to dietary source acts only by $10 \%$ of vitamin D sources.

Our study showed that $66.2 \%$ of deficient study population were with low level of indoor compared to $0 \%$ among optimal ones with odds ratio $(\mathrm{OR}=1.4)$ while regarding outdoor activity, $95.8 \%$ of deficient group were with low level compared to $44.4 \%$ of optimal one with odds ratio $(\mathrm{OR}=6.47)$. Moreover, The present study revealed that excess body fat, in the form of overweight and obesity is associated with an increased risk of suboptimal vitamin D status (29.6\%) of women having deficient vitamin D were 
overweight and obese compared to $(22.2 \%)$ of those who had normal level with odds ratio $(\mathrm{OR}=1.5)$ without any statistical significant difference.

These results are like the results of $\boldsymbol{E l}$ Rifai et al. (14) who reported that there was a significant moderate inverse correlation between BMI and maternal vitamin $\mathrm{D}$ level. Also, Agarwal, et al. (32) demonstrated that there was a strong correlation between increasing body mass index (BMI) and vitamin $\mathrm{D}$ deficiency and insufficiency. All obese females, i.e., $\mathrm{BMI} \geq 30$ were found to have vitamin $\mathrm{D}$ deficiency ( $100 \%$ prevalence).Moreover, in a Danish study, Andersen et al. ${ }^{(29)}$ found that vitamin D insufficiency during early pregnancy was positively correlated with higher pregnancy BMI.In addition,Perez-Lopezet $\quad \boldsymbol{a l l}^{\left({ }^{(2)}\right)}$ and $\boldsymbol{Y u}$ et al. ${ }^{(33)}$ supported the current results as they found thatamong pregnant women, maternal vitamin D values during first trimester of pregnancy have been negatively associated with maternal BMI,

On the other hand, Woonet al. (21) and Ekeroma et al. ${ }^{(30)}$ revealed thatthere were no associations between pre-pregnancy BMI with vitamin $\mathrm{D}$ deficiency. This difference may be attributed to different ethnic population, sampling size and technique, type of study and study setting.

\section{LIMITATIONS}

First, due to high cost of laboratory assessment of vitamin D level, we had a relatively small sample size.Second, some of cases had negative attitude and/or did not show much cooperation to answer the questionnaire.

\section{CONCLUSION}

Although Egypt is a country with abundant sunshine all year round, majority of pregnant females had vitamin D deficiency and insufficiency. Vitamin D deficiency had a role in spontaneous abortion especially recurrent spontaneous abortion.

By examining factors influencing vitamin D deficiency among pregnant females during early pregnancy, it was found that urban residence, nonworking status, low educational level; low socio economic status, white skin color and exposure to sun with more duration and moreexposed body surface area were protective factors for VDD while obesity, nullipara parity, less indoor and outdoor activity, more fish intake and less yogurt consumption were possible risk factors for VDD.

\section{RECOMMENDATION}

On the light of the previous results and discussion we recommend to implement a national strategy for screening, prevention, and treatment of vitamin D deficiency among females in different age group and to increase their awareness about the importance of vitamin D and unfavorable outcome of its deficiency on maternal and child life, ensure antenatal screening for VDD and nutritional education for sources of vitamin $\mathrm{D}$ and further research on large sample of population.

\section{REFERENCES}

1. Goddijn M, Christiansen OB, Elson Jet al. (2017): Recurrent pregnancy loss. Guideline of the European Society of Human Reproduction and Embryology. https://doi.org/10.1007/978-3-319-27452-2.

2. Royal College Obstetricians and Gynecologists (RCOG), (2011): The investigation and treatment of couples with recurrent first trimesterand second trimestermiscarriage. R Coll ObstetGynaecol., 17:118.

3. Salat-Baroux $\mathbf{J}(\mathbf{1 9 8 8})$ : Recurrent spontaneous abortions. ReprodNutr Dev., 28(6B):1555- 1568.

4. Abdi-Shayan S, Monfaredan A, Moradi Z, Oskoui MR, Kazemi T (2016): Association of CD46 IVS11724 C4G single nucleotide polymorphism in Iranian women with unexplained recurrent spontaneous abortion (URSA). Iran J Allergy Asthma Immunol., 15: 303-308.

5. Holick MF(2007):Vitamin D deficiency. $N$ Engl J Med., 357: 266-281.

6. Bikle D.D(2011):Vitamin D regulation of immune function. VitamHorm.,86: 1-21.

7. Lagishetty V, Liu NQ, Hewison M (2011):Vitamin D metabolism and innate immunity. MolCell Endocrinol., 347: 97-105.

8. Sowell K.D, Keen CL, Uriu-Adams JY(2015):Vitamin D and Reproduction: From Gametes to Childhood. Healthcare,3:1097-1120.

9. Lerchbaum E, Rabe T(2014):Vitamin D and female fertility. CurrOpinObstetGynecol., 26: 145-150.

10. Adams JS, Hewison M(2008): Unexpected actions of vitamin D: new perspectives on the regulation of innate and adaptive immunity. Nat ClinPractEndocrinolMetab., 4:80-90.

11. Ota K, Dambaeva S, Ae-Ra Han, Beaman Ket al. (2013): Vitamin D deficiency may be a risk factor for recurrent pregnancy losses by increasing cellular immunity and autoimmunity.Human Reproduction, 29: 208-219.

12. Nageshu N, KrishnaK, Krishna L, ShyamasundaraB et al. (2016): Study of prevalence of Vitamin D deficiency among pregnant women and its impact on feto maternal outcome. Int $\mathbf{J}$ Reprod Contracept Obstet Gynecol., 4(4):1174-118.

13. Botros RM, Sabry IM, AbdelbakyRS, Eid YA, Nasr MS, Hendawy LM (2015):Vitamin D 
deficiency among healthy Egyptian females.EndocrinolNutr., 62(7):314---321

14. El Rifai1NM, Abdel MoetyGA, GaafarHM, HamedDA (2013): Vitamin D deficiency in Egyptian mothers and their neonates and possible related factors.https://www.ncbi.nlm.nih.gov

15. Pereira-Santos M, Carvalho GQ, Couto RD et al.(2017): Vitamin D deficiency and associated factors among pregnant women of a sunny city in Northeast of Brazil. Nutr ESPEN., 23:240-244.

16. Aji A.S, Erwinda E, Yusrawati Y, Malik SG and Lipoe NI (2019): Vitamin D deficiency status and its related risk factors during early pregnancy: a cross sectional study of pregnant Minangkabau women, Indonesia. BMC Pregnancy and Childbirth, 19:183

17. Gonçalves D.R, Braga A, Braga A, Marinho A (2018): Recurrent pregnancy loss and vitamin D: A review of the literature. Am J Reprod Immunol., 80:e13022.

18. WHO (2015): Body mass index.http://www.euro.who.int/en/healthtopics/disease-prevention/nutrition/a-healthylifestyle/body-mass-index-bmi.

19. El Koumi MA, Ali YF, Abd El Rahman RN (2013):Impact of maternal vitamin D status during pregnancy on neonatal vitamin D status. The Turkish Journal of Pediatrics, 55: 371-377

20. Hussein I, Taha Z, Tewfik I, Badawi S, Siddieg H et al. (2016): Risk Factors for Maternal Vitamin D Deficiency within the United Arab Emirates. J Preg Child Health, 3:5

21. WoonFC, Chin YS, Ismail IH et al. (2019): Vitamin $\mathrm{D}$ deficiency during pregnancy and its associated factors among third trimester Malaysian pregnant women. PLoSOne.J., 14(6):e0216439.

22. Hou W, Yan X-t, Bai C-m, Zhang X-w, Hui L-y, Yu X-w (2016): Decreased serum vitamin D levels in early spontaneous pregnancy loss. European Journal of Clinical Nutrition,70: 1004-1008

23. Ghaedi N, Forouhari S, Zolghadri J, Sayadi Met al.(2016): Vitamin D deficiency and recurrent pregnancy loss in Iranian women. Global Advanced Research Journal of Medicine and Medical Sciences,5(6) : 194-198.
24. Andersen LB, Jørgensen JS, Jensen TK, Dalgård C, Barington T, Nielsen $\mathbf{J}$ et al.(2015):Vitamin D insufficiency is associated with increased risk of firsttrimester miscarriage in the Odense Child Cohort. Am J Clin Nutr., 102: 633-638.

25. Ozkan S, Jindal S, Greenseid K, Shu J, Zeitlian G, Hickmon C and Pal L (2010):Replete vitamin D stores predict reproductive success following in vitro fertilization. Fertil. Steril., 94:1314-1319.

26. Møller UK, Streym S, Heickendorff L, Mosekilde L, Rejnmark L (2012): Effects of 25OHD concentrations on chances of pregnancy and pregnancy outcomes: a cohort study in healthy Danish women. Eur J Clin Nutr., 66: 862-868. 35

27. Flood-Nichols S, Tinnemore D, Huang $\mathbf{R}$, Napolitano P, Ippolito DL (2015):Vitamin D deficiency in early pregnancy. PLoS ONE, 10: e0123763.

28. Perez-Lopez F, Fernandez-Alonso A, FerrandoMarco P, Gonzalez-Salmeron M et al. (2011): First trimester serum 25-hydroxyvitamin D status and factors related to lower levels in gravid living in the Spanish Mediterranean coast. Reproductive sciences , 18:730-736.

29. Andersen L, Abrahamsen B, Dalgard C, Kyhl H, Beck-Nielsen Set al.(2013):Parity and tanned white skin as novel predictors of vitamin D status in early pregnancy: A population-based cohort study.Clinical Endocrinology, 79: 333-341,

30. Ekeroma A, Camargo CA, Robert S, Clare Wet al. (2015):Predictors of vitamin D status in pregnant women in New Zealand.https://www.nzma.org.nz >

31. Bukhary N.I, Zaleha MI, Khadijah S, Khor GL, Zaleha AM, Haslinda Het al. (2016): Risk factors for antenatal hypovitaminosis $\mathrm{D}$ in an urban district in Malaysia. BMC Pregnancy Childb., 16(1):156.

32. Agarwal1 S, ChaudharyM, Chauhan1 J, Vacchani A(2016): Assessment of vitamin D deficiency in pregnant females attending antenatal care clinic at tertiary care hospital. International Journal of Scientific Study, 4:5.

33. Yu CK, Ertl R, Samaha R, Akolekar R, Nicolaides K (2011): Normal range of maternal serum vitamin $D$ at 11-13 weeks' gestation. Fetal diagnosis and therapy, 30:94-99. 